\title{
Constrained annealing for spin glasses
}

\author{
Giovanni Paladin ${ }^{1}$, Michele Pasquini ${ }^{1}$, and Maurizio Serva ${ }^{2}$ \\ ${ }^{1}$ Dipartimento di Fisica, Università dell'Aquila \\ I-67100 Coppito, L'Aquila, Italy \\ ${ }^{2}$ Dipartimento di Matematica, Università dell'Aquila \\ I-67100 Coppito, L'Aquila, Italy
}

\begin{abstract}
The quenched free energy of spin glasses can be estimated by means of annealed averages where the frustration or other self-averaging variables of disorder are constrained to their average value. We discuss the case of $d$-dimensional Ising models with random nearest neighbour coupling, and for $\pm J$ spin glasses we introduce a new method to obtain constrained annealed averages without recurring to Lagrange multipliers. It requires to perform quenched averages either on small volumes in an analytic way, or on finite size strips via products of random transfer matrices. We thus give a sequence of converging lower bounds for the quenched free energy of $2 d$ spin glasses.
\end{abstract}

PACS NUMBERS: 05.50.+q, 02.50.+s, 75.10.N 


\section{Introduction}

Disordered systems are made of random variables which relax to equilibrium on very different time scales. For instance in the standard models of spin glass, the spins arrange themselves to minimize the free energy while the random couplings are assumed to be frozen in their initial values, since their evolution time is much longer. As a consequence, one should consider different kinds of averages: quenched averages for the frozen variables, and annealed averages for the fast variables. However, it is much easier to compute annealed averages, which give lower bounds of the quenched free energy, the physically relevant quantity. These bounds are often very poor estimates, since in annealed averages the main contribution comes from a set of disorder realizations which have zero probability in the thermodynamic limit. In those realizations, the quenched random variables are arranged in order to minimize the free energy, at difference with a typical sample of the system where they are frozen in a given realization.

The idea of constrained annealing is to perform annealed averages where the random variables are partly frozen by the requirement of satisfying appropriate constraints, related to some disorder self-averaging variables. The standard way to impose a constraint is by a Lagrange multiplier. By that method, the constrained annealed average allows one to introduce a sort of Gibbs thermodynamic potential, depending on the temperature and on the Lagrange multipliers which play the role of the chemical potential in ordinary statistical mechanics [1]. In the thermodynamic limit, the values of the multipliers that maximize the Gibbs potential are those ones that select the realizations with a correct value of the disorder intensive variables, and, at the same time, that minimize the difference between the mean free energy density and its annealed approximations. 
Constrained annealing for disordered systems has been proposed and applied in particular contexts by many authors (see [2], [3], [4], [5], [6]). A general formulation of the method and its physical meaning can be found in [1].

The purpose of this paper is to show that it is possible to perform constrained annealed averages without Lagrange multipliers, in $d$-dimensional spin glasses with dichotomic random couplings in terms of a quenched average over a finite size subsystem . This is often possible either in an analytic or in a numeric way. Using our method, we are able to obtain very good lower bounds of the quenched free energy of two dimensional spin glasses.

The summary of the paper is the following:

In section 2 , we recall some basic facts about spin glasses. We also classify the set of the constraints necessary to recover the quenched free energy by annealed averages. In section 3, we study the Ising model with couplings which can assume the values $J_{i j}= \pm 1$ with equal probability ( the so-called $\pm J$ spin glass). In section 4 , we introduce our method to obtain constrained annealed averages without recurring to Lagrange multipliers in the $\pm J$ spin glass. In section 5 , we apply the method to loops of infinite length using the Lyapunov exponent of product of random transfer matrices. We are thus able to get accurate estimates of the free energy in two dimensions.

\section{Constraints for $d$-dimensional spin glasses}

Spin glasses are the challenge of constrained annealing. In that case, the relevant variables to be constrained should be related to frustration [7], as first remarked by Toulouse and Vannimenus in 1976 [5]. As far as we know, there are only Montecarlo simulations, see e.g. [8], [9], which used that idea while no analytic calculations have been performed on that ground. However, it is possible to get analytic results 
in dimension $d \geq 2$ with constraints connected to frustration. Let us consider $d$ dimensional Ising models with nearest neighbour couplings $J_{i j}$ which are independent identically distributed (i.i.d.) random variables, in absence of external magnetic field. The partition function is

$$
Z_{N}=\sum_{\{\sigma\}} \prod_{i, j} \exp \left\{\beta J_{i j} \sigma_{i} \sigma_{j}\right\}
$$

where the product runs over $i, j$ which are nearest neighbours. Using standard relations of the hyperbolic functions, (2.1) becomes

$$
Z_{N}=\sum_{\{\sigma\}} \prod_{i, j} \cosh \left(\beta J_{i j}\right)\left[1+\sigma_{i} \sigma_{j} \tanh \left(\beta J_{i j}\right)\right]
$$

This form is useful since shows that the non-trivial part of the partition function is given by $\prod_{i, j}\left[1+\sigma_{i} \sigma_{j} \tanh \left(\beta J_{i j}\right)\right]$. A typical term in that product is $\sigma_{i} \sigma_{j} \sigma_{j} \sigma_{l} \cdots \tanh \left(\beta J_{i j}\right) \tanh \left(\beta J_{j l}\right) \cdots$, where the bonds $\{i, j\},\{j, l\} \cdots$ can form either a closed or an open line on the lattice. If the bonds form an open line, at least two distinct spin variables survive as factor of the product of the various $\tanh \left(\beta J_{i j}\right)$, and the related term gives a zero contribution to the partition function, after performing the sum over the $\sigma$ configurations. As a consequence, one has to consider only the terms corresponding to bonds which form a closed line (where the product of the $\sigma$ 's is equal to unity since they appear twice) so that (2.2) can be written as a sum over all the possible loops $L_{s}^{(r)}$, where $r \geq 4$ indicates the length of the loop ( $r$ has to be even) and $s$ labels different loops with the same length:

$$
Z_{N}=e^{\sum_{i, j} \ln \cosh \left(\beta J_{i j}\right)} 2^{N}\left(1+\sum_{\left\{L_{s}^{(r)}\right\}} \prod_{\{i, j\} \in L_{s}^{(r)}} \tanh \left(\beta J_{i j}\right)\right)
$$

It follows that in a system with a finite number $N$ of spins, the free energy of a disorder realization is given by

$$
y_{N} \equiv-\frac{1}{\beta N} \ln Z_{N}=-\frac{1}{\beta}\left(B+\widetilde{F}_{N}\left(t^{(4)}, \cdots, t^{(R)}\right)\right)
$$


where $R$ is the maximum possible length of a loop in the system $(R<\operatorname{const} N)$, and

$$
B=\ln 2+\frac{1}{N} \sum_{i, j} \ln \cosh \left(\beta J_{i j}\right)
$$

and

$$
\widetilde{F}_{N}\left(t^{(4)}, \cdots, t^{(R)}\right)=\frac{1}{N} \ln \left(1+\sum_{\left\{L_{s}^{(r)}\right\}} t_{s}^{(r)}\right)
$$

This function depends on the new set of variables

$$
t_{s}^{(r)}=\prod_{\{i, j\} \in L_{s}^{(r)}} \tanh \left(\beta J_{i j}\right)
$$

given by products which run on the loops $L_{s}^{(r)}$, i.e. plaquettes of larger and larger perimeter at increasing $r$. For instance, the loop $L^{(4)}$ is the elementary plaquette of four bonds. Let us stress that a loop $L^{(r)}$ can be built up as a set of two (or more) distinct and separate loops $L^{\left(r_{1}\right)}$ and $L^{\left(r_{2}\right)}$ with $r_{1}+r_{2}=r$. In the following we often label a coupling by only one label instead of two ones, i.e. $J_{k l}$ is denoted by $J_{k}$, since there is no risk of ambiguity on a closed line.

As the number of the nearest neighbour couplings in a hypercubic $d$-dimensional lattice is $d N$, in the thermodynamic limit the quenched free energy reads

$$
f \equiv \lim _{N \rightarrow \infty} \overline{y_{N}}=-\frac{1}{\beta}\left[d \overline{\ln \cosh \left(\beta J_{i j}\right)}+F+\ln 2\right]
$$

where

$$
F=\lim _{N \rightarrow \infty} \frac{1}{N} \overline{\ln \left[1+\sum_{\left\{L_{s}^{(r)}\right\}} t_{s}^{(r)}\right]}
$$

The reduction of the partition function (2.1) into (2.3) is the procedure used in the Kac and Ward solution of the $2 d$ Ising model without disorder.

$\mathrm{Eq}(2.5)$ is fundamental since it shows that the quenched free energy depends only on the distributions of the variables $t^{(r)}$, apart the mean value $\overline{\ln \cosh \left(\beta J_{i j}\right)}$. The 
relevant variables to be fixed to their mean value in a constrained annealed average of the free energy are related, with increasing difficulty

1) $\ln \cosh \beta J_{i j}$

2) $t^{(4)}=\tanh \left(\beta J_{1}\right) \tanh \left(\beta J_{2}\right) \tanh \left(\beta J_{3}\right) \tanh \left(\beta J_{4}\right)$

3) $t^{(r)}$ with $r \geq 6$ (considering only connected loops).

Fixing all the constraints corresponding to those quantities in an annealed average is equivalent to solve the system with quenched disorder. This can be understood noting that in (2.6) we have a sum over all the possible $t_{s}^{(r)}$. Because of the law of the large numbers the rescaled sum of the $t$-variables corresponding to the same topological kind of loops self-averages to its quenched mean value. Moreover, in the appendix we shall prove that this result holds even in a finite volume system for a $\pm J$ spin glass: the quenched free energy can be obtained by a minimization of the logarithm of the annealed average of a Grand-partition function with respect to the Lagrange multipliers related to the variables $t_{s}^{(r)}$ over all the possible loops of the finite system. For a generic distribution of the couplings, an extension of this statement is possible.

In one dimension there are no loops, so that the constraint related to point 1) is sufficient to obtain the exact solution. In general, we expect that many physical features of a spin glass can be reproduced already by considering the first two points.

The case of random couplings $J_{i j}= \pm 1$ with equal probability is particularly simple in this respect, since the variable $\ln \cosh \beta J_{i j}$ is a constant and thus can be ignored. Moreover, one immediately sees that (2.4c) becomes

$$
t_{s}^{(r)}=\tanh ^{r}(\beta) \prod_{\{i, j\} \in L_{s}^{(r)}} J_{i j}
$$

implying that the quenched free energy only depends on the values of the product of 
$J_{i j}$ on closed lines of bonds. The non trivial part of the free energy $y_{N}$ in (2.4) is thus

$$
\widetilde{F}_{N}=\frac{1}{N} \ln \left(1+x_{4} \tanh ^{4}(\beta)+x_{6} \tanh ^{6}(\beta)+\cdots\right)
$$

where the coefficients $\left\{x_{4}, x_{6}, \cdots\right\}$ are non-independent random quantities. As a consequence, the quenched free energy is

$$
f=-\frac{1}{\beta}\left(d \ln \cosh \beta+\ln 2+\lim _{N \rightarrow \infty} \frac{1}{N} \overline{\ln \left(1+x_{4} \tanh ^{4}(\beta)+x_{6} \tanh ^{6}(\beta)+\cdots\right)}\right)
$$

In the annealed model, the last non-trivial term of (2.9) vanishes since $\overline{x_{i}}=0$ for all $i$ 's (each $x_{i}$ is a sum of products of independent random couplings). In the limit of high temperature $(\beta \rightarrow 0)$, the difference between annealed free energy and quenched free energy thus is of order $\tanh ^{4} \beta \sim \beta^{4}$. More important, the simplest relevant constraint in the $\pm J$ spin glass is the frustration $\widetilde{J}_{p}$ on the elementary square plaquette of four couplings $J_{1}, J_{2}, J_{3}, J_{4}$, that is

$$
\widetilde{J}_{p}=-J_{1} J_{2} J_{3} J_{4}
$$

since $t^{(4)}=-\widetilde{J}_{p} \tanh ^{4} \beta$.

\section{3. $\pm J$ spin glasses}

In this section we consider a $d$-dimensional spin glass with i.i.d. nearest neighbour couplings which assume two possible value $J_{i j}= \pm 1$ with equal probability. From (2.9), one sees that the annealed free energy density is

$$
f_{a}=-\frac{1}{\beta}(\ln 2+d \ln \cosh \beta)
$$

In one dimension, there are no loops and $f_{a}$ is equal to the quenched free energy density $f$, since $\ln \cosh \beta J_{i j}$ is a constant, at difference with the gaussian case where 
that variable is random and has to be constrained in order to recover $f[10]$. In section 2, we have recalled standard results which show that it is necessary to constrain variables associated to the loop variable $t^{(4)}$, in order to improve (3.1). As first noted by Toulouse and Vannimenus [5], one has to consider the intensive variable

$$
\alpha_{N}=\frac{1}{N} \sum_{\left\{L_{s}^{(4)}\right\}} \widetilde{J}_{p}
$$

with has zero mean value, $\overline{\alpha_{N}}=0$. Let us remark that it is a self-averaging quantity, that is $\lim _{N \rightarrow \infty} \alpha_{N}=0$ for almost all disorder realizations. The related constrained annealed average can be regarded as the Grand-partition function

$$
\Omega_{N}(\beta, \mu) \equiv \overline{Z_{N} e^{-N \mu \alpha_{N}}}
$$

In the thermodynamic limit the Lagrange multiplier $\mu$ that maximizes

$$
g(\beta, \mu)=-\lim _{N \rightarrow \infty} \frac{1}{\beta N} \ln \Omega_{N}(\beta, \mu)
$$

fixes the frustration on square plaquettes to its mean value. Moreover it is simple to show the following inequality:

$$
f \geq f_{a c}^{s q} \equiv \max _{\mu} g(\beta, \mu)
$$

In our case, we have to compute

$$
\Omega_{N}(\beta, \mu)=\overline{\sum_{\{\sigma\}} \exp \left\{\sum_{i, j} \beta J_{i j} \sigma_{i} \sigma_{j}-\sum_{\left\{L_{s}^{(4)}\right\}} \mu \widetilde{J}_{p}\right\}}
$$

Taking into account that the couplings are dichotomic with equal probability, one can perform the gauge transformation $J_{i j} \rightarrow J_{i j} \sigma_{i} \sigma_{j}$ which leaves unchanged the free energy of a disorder realization of the system as well as $\widetilde{J}_{p}$, i.e. $\widetilde{J}_{p} \rightarrow \widetilde{J}_{p}$. As a consequence, (3.3) becomes

$$
\Omega_{N}(\beta, \mu)=2^{N} \overline{\exp \left\{\sum_{i, j} \beta J_{i j}-\sum_{\left\{L_{s}^{(4)}\right\}} \mu \widetilde{J}_{p}\right\}}
$$


This expression (which is meaningful for any dimension $d \geq 2$ ) cannot be computed exactly including all the possible square plaquettes $L_{s}^{(4)}$ of the $d$-dimensional lattice. It can be shown that the problem is equivalent to solve a $d$-dimensional gauge model without disorder, whose solution is not known for $d \geq 2$.

However, an exact solution can be achieved if we restrict the sum (3.4) only on a part (of order $N$ ) of the plaquettes. In other terms the constraint is imposed over a fraction of the plaquettes, that is we are able to partially fix the plaquette frustration to its mean value. As a consequence, the annealed system exhibits a 'residual' frustration related to the non-constrained plaquettes.

Let us consider the $2 d$ model. It is convenient to limit ourselves to consider one half of the plaquettes, which must be chosen in such a way that they do not share any coupling, as it happens for the black squares of a chessboard. With this restriction (3.4) contains a sum over products of $N / 2$ independent variables (corresponding to the $N / 2$ black plaquettes) and the Grand-partition function can be rewritten as:

$$
\Omega_{N}(\mu)=2^{N}\left(\overline{\exp \left\{\beta\left(J_{1}+J_{2}+J_{3}+J_{4}\right)+\mu J_{1} J_{2} J_{3} J_{4}\right\}}\right)^{\frac{N}{2}}
$$

where $J_{1}, J_{2}, J_{3}, J_{4}$ are the couplings of one of the plaquettes. After maximizing with respect to $\mu$ the Gibbs potential [1], one obtains

$$
f_{a c}^{s q}=-\frac{1}{\beta}\left\{\frac{1}{2} \ln 2+\frac{1}{4} \ln \cosh 2 \beta+\frac{1}{4} \ln (3+\cosh 4 \beta)\right\}
$$

The functions $f_{a}$ and $f_{a c}^{s q}$ of the $2 d$ spin glass are shown in fig 1 . At difference with the annealed case the entropy of the constrained annealed system never becomes negative. We have thus obtained a real qualitative improvement.

It is also interesting to consider the heat capacity $C$ which can be explicitly computed. For $\beta \rightarrow \infty$ one has

$$
C \sim \beta^{2} e^{-4 \beta}
$$


The argument of the exponential has been conjectured in [11] for the quenched model and it is different from the exact one-dimension result where the argument is $-2 \beta$. Note that the annealed system also gives $C \sim \exp (-2 \beta)$.

Finally, since we have fixed only one half of the single plaquette frustration, it is reasonable to ask what is the residual frustration $\phi_{a c}$. Since the black plaquettes have zero frustration, this quantity will be one half of the frustration of a white plaquette. The four couplings forming a white plaquette are independent since they are not coupled by the Lagrange multiplier. Therefore, the white plaquette frustration can be written as $-<<J_{i}>>^{4}$, and the global frustration $\phi_{a c}$ reads:

$$
\phi_{a c}=-\frac{1}{2}<<J_{i}>>^{4}
$$

where $\langle<\cdot>>$ is an average on the Gibbs measure associated to the Grand-partition function (3.3). One has therefore

$$
<<J_{i}>>=-\frac{1}{2} \frac{\partial\left(\beta g\left(\beta, \mu^{*}\right)\right)}{\partial \beta}
$$

where $\mu^{*}$ is the value which maximizes $g(\beta, \mu)$, and the factor $1 / 2$ stems from the fact that the total number of couplings is $2 N$. Since

$$
f_{a c}^{s q}(\beta)=g\left(\beta, \mu^{*}(\beta)\right)
$$

and since the derivative with respect to $\mu$ vanishes, one has

$$
<<J_{i}>>=-\frac{1}{2} \frac{d\left(\beta f_{a c}^{s q}\right)}{d \beta}
$$

Using the expression (3.6) for $f_{a c}^{s q}$ we can compute the residual frustration

$$
\phi_{a c}=-\frac{1}{2}<<J_{i}>>^{4}=-\frac{1}{2}\left[\frac{\operatorname{th}(2 \beta)}{4}+\frac{\operatorname{th}(2 \beta)}{4-2 \operatorname{th}^{2}(2 \beta)}\right]^{4}
$$

At zero temperature $\phi_{a c}=3^{4} / 2^{9} \approx 0.158$. This result indicates the presence of a small but non vanishing residual frustration. An analogous expression can be found from 
the annealed free energy $f_{a}$. In this case all plaquettes can have negative frustration and one has

$$
\phi_{a}=-<<J_{i}>>^{4}=-\operatorname{th}^{4}(\beta)
$$

where the average is in the Gibbs measure generated by the annealed average. The frustration $\phi_{a}(T=0)=-1$ since at zero temperature the spins are aligned and the annealed system is completely ferromagnetic. Both $\phi_{a}$ and $\phi_{a c}$ are reported in Fig 2. The fact that in the constrained annealed model the residual frustration does not vanish indicates that the free energy estimate could be improved by further constraints as we shall discuss in the next section.

\section{Constraints without Lagrange multipliers}

As far as we know, up to now constrained annealing has been performed only using Lagrange multipliers. This is a strong limitation, since in most cases it is too difficult to derive analitically an annealed average using more than one constraint. The spin glass with dichotomic couplings has some algebraic features which permit us to introduce an alternative method, in order to obtain constrained annealed averages avoiding in this way the problem of the minimization of the Lagrange multipliers.

Let us consider a $d$-dimensional lattice of $N$ points: the total number of the couplings is $d N$, but the system can be fully described by a lower number of independent random variables. In fact the non-trivial part of the quenched free energy (2.6) is a function of the variables $\left\{t_{s}^{(r)}\right\}$, see $(2.4 \mathrm{c})$, defined on the closed loops of the

lattice. Indeed, from a topological point of view any closed loop $L_{s}^{(r)}$ can be thought as the union of some elementary square plaquettes, say a number $k$, so that the related 
variable $t_{s}^{(r)}$ can be written as:

$$
t_{s}^{(r)}=\tanh ^{r}(\beta) \prod_{i=1}^{k} \widetilde{J}_{p}^{(i)}
$$

where we have introduced the frustrations $\widetilde{J_{p}}=\prod_{i=1}^{4} J_{i}= \pm 1$ with equal probability of the $k$ plaquettes (the index $i$ runs over the four sites of each square). Let us stress that a loop can be either connected or disconnected, i.e. it is not necessary that the plaquettes are neighbours. Notice that the plaquette frustration was previously defined with a minus sign (see $(2.10)$ ), but in this context we neglect it to simplify the notation. Moreover, we dot not assume periodic boundary conditions in this section.

In other terms, we can consider the plaquette frustrations as the random variables of the system, instead of the couplings, with no loss of generality. We shall see that the number of frustrations necessary for a full description of the system is always lower than $d N$, the total number of couplings. For instance, in $2 d$, there are $2 N$ couplings, while the number of plaquette frustrations is of order $N$ when $N \rightarrow \infty$.

However, the $2 d$ case exhibits a special feature, since all the possible plaquette frustrations turn out to be a set of independent random variables (let us recall that we do not assume periodic boundary condition). In the general case $d \geq 3$, this statement is no longer valid. Indeed, let us consider a cube in a $d$-dimensional lattice: a moment of reflection shows that the product of the related six plaquette frustrations has to be 1 , so that only five frustrations are independent random variables. As a consequence, for $d \geq 3$ the change of random variables from the couplings to the plaquette frustrations involves only a subset of them, let us say $N^{*}$, chosen in such a way that they are independent random variables and that they can build up every possible closed loop on the lattice.

Let us illustrate this change of random variables for a $3 d$ lattice of $N$ points, where we can introduce three integer coordinates $\{x, y, z\}$. A square plaquette has 
three different orientations, belonging to a $x=$ const plane, or $y=$ const, or $z=$ const. In the thermodynamic limit one has $N^{\frac{1}{3}}$ planes for each orientation, and every plane is a set of $N^{\frac{2}{3}}$ plaquettes, for a total number of $3 N$ plaquettes in the whole lattice.

Consider a cube of six plaquettes, with one of them on the $z=0$ plane. As previously discussed, one of the $\widetilde{J}$ 's cannot belong to the subset of plaquette frustrations that describe the whole system, let us say the frustration of the plaquette on the $z=1$ plane. Let us now consider the other cube sharing this 'neglected' plaquette. By introducing as random variables of the system the frustrations which are related to the four plaquettes that share a coupling with the plaquette on the $z=1$ plane, we have to neglect the sixth frustration of the plaquette on the $z=2$ plane, since the product of all the plaquette frustrations of these two nearest neighbour cubes is fixed to 1 (the presence of the plaquette on $z=1$ in the product is irrelevant, since it appears twice). These arguments can be easily repeated, so that the subset of plaquette frustrations that fully describes the whole system can be found as follows: one has to consider two distinct orientations and to take into account all the plaquettes with these orientations, for a total number of $2 N$. Moreover, the third orientation gives only a plane of $N^{\frac{2}{3}}$ plaquettes (the $z=0$ plane in our example), so that in the thermodynamic limit the number of independent random frustrations $N^{*}$ that fully describe the systems grows as $2 N$.

The generalization of this result is immediate: in the thermodynamic limit the $d$ dimensional lattice of $N$ points (with $d \geq 3$ ) is built up by $N\left(\begin{array}{l}d \\ 2\end{array}\right)$ distinct plaquettes, since $\left(\begin{array}{l}d \\ 2\end{array}\right)$ is the number of different orientations for a plane in the lattice, but only a number $N^{*}=2 N$ of plaquette frustrations is necessary to describe the system. In fact the change of random variables involves only the frustrations of the plaquettes with two well-defined orientations ( $2 N$ plaquettes), together with a plane of plaquettes for each 
one of the other orientations, but in the thermodynamic limit the main contribution to $N^{*}$ comes from the former term. Using this subset of plaquettes, whose related frustrations are all independent random variables, it is possible to build up every possible closed loop on the $d$-dimensional lattice.

In conclusion the described change of variables permits us to reduce the number of independent random variables necessary to describe the system, from $d N$ couplings to $N^{*}$ frustrations, with $N^{*}=N$ for $d=2$ or $N^{*}=2 N$ for $d \geq 3$. In the following, if not differently specified, the term 'frustration' always indicates one of the $N^{*}$ random variables of the system.

Recalling (4.1), equation (2.6) becomes:

$$
F=\lim _{N \rightarrow \infty} \frac{1}{N} \overline{\ln \left[1+\sum_{k=1}^{N^{*}} \sum_{i_{1} \ldots i_{k}} \widetilde{J}_{p}^{\left(i_{1}\right)} \ldots \widetilde{J}_{p}^{\left(i_{k}\right)} \tanh ^{r\left(i_{1}, \ldots, i_{k}\right)}(\beta)\right]}
$$

where one considers all the products of $k \in\left[1, N^{*}\right]$ elementary plaquettes ( the indices $i_{1} \neq i_{2} \neq \ldots \neq i_{k}$ run from 1 to $\left.N^{*}\right)$ and $r\left(i_{1}, \ldots, i_{k}\right)$ is the length of the loop built up by the $k$ plaquettes, while the overline represents now the average over the frustrations.

In the following paragraph, we consider the plaquettes of the lattice only from a topological point of view, i.e., the fact that a frustration of a plaquette is a random variable of the system (or not) is irrelevant. At this point, we perform a decomposition of the set of plaquettes into subsets such that each coupling $J_{i j}$ belongs to one and only one of these subsets. It follows that two distinct subsets can have in common only isolated lattice points. For instance, three different decompositions of the bonds of a $2 d$ lattice (the black regions) are illustrated in fig 3. It is worth stressing that, after the decomposition, we get a collection of sub-systems which do not cover the whole original lattice, e.g. in fig $3 \mathrm{a}$ only one half of the $2 \mathrm{~d}$ lattice is covered. This kind of decomposition divides the plaquettes into two classes: the 'black' ones, which 
are organized in groups corresponding to the sub-systems (e.g. the crosses in fig $3 \mathrm{~b}$ ), and the 'white' ones, which do not belong to any sub-system.

Coming back to the frustrations, note that in general there are many 'white' or 'black' plaquettes whose frustration is not a random variable of the system.

At this point we can derive an upper estimate $F_{a c}$ of the function (4.2), by treating all the random variables of frustration associated to the 'white' plaquettes as annealed variables, i.e. by averaging over these variables only the argument of the logarithm in (4.2), instead of the logarithm itself:

$$
F_{a c}=\lim _{N \rightarrow \infty} \frac{1}{N} \overline{\ln \left[1+\sum_{k=1}^{N^{*}} \sum_{i_{1} \ldots i_{k}}{\overline{\widetilde{J}_{p}^{\left(i_{1}\right)} \ldots \widetilde{J}_{p}^{\left(i_{k}\right)}}}^{(w)} \tanh ^{r\left(i_{1}, \ldots, i_{k}\right)}(\beta)\right]}
$$

where $\boldsymbol{-}^{(w)}$ and $-^{(b)}$ represent the averages over the frustrations related to, respectively, the 'white' and the 'black' squares. In (4.3) only the terms $\widetilde{J}_{p}^{\left(i_{1}\right)} \ldots \widetilde{J}_{p}^{\left(i_{k}\right)}$ with all 'black' frustrations do not vanish after performing the 'white' average:

$$
F_{a c}=\lim _{N \rightarrow \infty} \frac{1}{N}{\overline{\ln \left[1+\sum_{k=1}^{N_{b}} \sum_{i_{1} \ldots i_{k}} \widetilde{J}_{p}^{\left(i_{1}\right)} \ldots \widetilde{J}_{p}^{\left(i_{k}\right)} \tanh ^{r\left(i_{1}, \ldots, i_{k}\right)}(\beta)\right]}}^{(b)}
$$

where now the indices $i_{1}, \ldots, i_{k}$ run only over the $N_{b}$ 'black' frustrations. It should be remarked that after performing the 'white' average, the surviving loops do not connect different sub-systems. In other terms, any loop appearing in the average (4.4) can be decomposed into a set of 'sub-loops', each of them limited to a single sub-system. This implies that $\tanh ^{r\left(i_{1}, \ldots, i_{k}\right)}(\beta)$ can be factorized, i.e., $r\left(i_{1}, \ldots, i_{k}\right)$ is the sum of the lengths of the various 'sub-loops'. As a consequence, the whole argument of the logarithm in (4.4) is factorized among all the sub-systems, and it immediately follows:

$$
F_{a c}=\frac{d}{n_{j}} \overline{\ln \left[1+\sum_{k=1}^{n_{p}} \sum_{i_{1} \ldots i_{k}} \widetilde{J}_{p}^{\left(i_{1}\right)} \ldots \widetilde{J}_{p}^{\left(i_{k}\right)} \tanh ^{r\left(i_{1}, \ldots, i_{k}\right)}(\beta)\right]}
$$

where the average is performed over the $n_{p}$ 'black' plaquette frustrations that belong to a single sub-system, and where $n_{j}$ is the number of couplings in the sub-system, so that 
$\frac{d}{n_{j}}$ represents the total number of sub-systems, rescaled with $N$ in the thermodynamic limit. Recalling (2.5), from (4.5) the lower estimate $f_{a c}$ of the quenched free energy yields:

$$
-\beta f_{a c}=d \ln \cosh (\beta)+\frac{d}{n_{j}} \overline{\ln \left[1+\sum_{k=1}^{n_{p}} \sum_{i_{1} \ldots i_{k}} \widetilde{J}_{p}^{\left(i_{1}\right)} \ldots \widetilde{J}_{p}^{\left(i_{k}\right)} \tanh ^{r\left(i_{1}, \ldots, i_{k}\right)}(\beta)\right]}+\ln 2
$$

It is preferable to choose a topological decomposition of the system such that the $n_{p}$ 'black' frustrations fully describe the single sub-system. In this context $F_{a c}$ is practically equivalent to the quenched free energy of the sub-system, a part a factor proportional to $\ln \cosh \beta$. If $n_{p}$ is not too large, the computation of $F_{a c}$ can be performed analitically or numerically.

The correct multiplicative factors to transform the quenched free energy of a subsystem to the global constrained free energy (4.6) can also be obtained by a simple argument. Indeed the partition function $Z_{n_{s}}$ of a sub-system made of $n_{s}$ spins and $n_{j}$ couplings is the sum of $2^{n_{s}}$ terms which are given by the product of $n_{j}$ exponentials while the global constrained Grand-partition function $\Omega_{N}$ of a system with $N$ spins and $d N$ couplings is the sum of $2^{N}$ terms which are given by the product of $d N$ exponentials. In order to compare two quantities of order one, one has to write the following equality:

$$
\left(2^{-N} \Omega_{N}\right)^{\frac{1}{d N}}=\left(2^{-n_{s}} Z_{n_{s}}\right)^{\frac{1}{n_{j}}}
$$

so that

$$
f_{a c}=-\frac{1}{\beta}\left(1-d \frac{n_{s}}{n_{j}}\right) \ln 2+d \frac{n_{s}}{n_{j}} f_{s u b} .
$$

This formula is completely equivalent to (4.6), but has the advantage that the quenched free energy $f_{\text {sub }}$ of the sub-system explicitly appears. The ground state energy can be estimated by the saddle point method when $\beta \rightarrow \infty$ in the quenched average of $Z_{n_{s}}$ :

$$
E_{a c}(T=0)=\frac{d}{n_{j}} \overline{\max _{\{\sigma\}} H(\{J\})}
$$


where the maximum is taken over all the $\sigma$ configurations for each disorder realization. One can also obtains the residual entropy as

$$
S_{a c}(T=0)=\left(1-\frac{n_{s}}{n_{j}} d\right) \ln 2+\overline{\ln \operatorname{deg}(\{J\})}
$$

where $\operatorname{deg}(\{J\})$ is the number of disorder configurations which have energy $H(\{J\})$ equal to $E_{a c}(T=0)$.

Let us briefly resume the discussion, in order to clarify the meaning of the result. Using a topological decomposition of the system into independent sub-systems, we have derived a lower estimate of the quenched free energy that depends from the quenched free energy of the single sub-system. As discussed in the appendix, the quenched free energy of a sub-system can be obtained by a minimization of an annealed average of the Grand-partition function with Lagrange multipliers over all its possible loops. Using our procedure, we are able to get the annealed average $f_{a c}$ of the global system where the constraints are imposed over all the possible rescaled sums of frustration on all the loops that appear in the various sub-systems.

To illustrate our method, we apply it in the case of sect 3, i.e. the decomposition of a $2 d$ spin glass in 'white' and 'black' square plaquettes, like in a chessboard, see fig 3a. In this case all the $N$ plaquette frustrations represent a set of independent random variables, i.e. $N^{*}=N$. One half of them is related to 'white' plaquettes, so that they are treated as annealed variables, while the other half related to 'black' plaquettes as quenched variables. We also have $n_{j}=4$ and $n_{p}=1$, so that (4.6) becomes:

$$
-\beta f_{a c}^{s q}=2 \ln \cosh (\beta)+\frac{1}{4} \ln \left[1-\tanh ^{8}(\beta)\right]+\ln 2
$$

It is easy to check that after trivial algebraic manipulations one gets again (3.6).

The ground state energy and the zero temperature entropy can be directly computed from (4.10), and respectively are:

$$
E_{a c}^{s q}(T=0)=-1.5 \quad S_{a c}^{s q}(T=0)=0
$$


The method of constrained annealed averages without multipliers can be applied to non-elementary sub-systems in an easy numerical way, since one has not to introduce a set of corresponding Lagrange multipliers. In order to improve (4.10) and (4.11), we have considered a partition of the $2 d$ lattice into independent crosses of $n_{p}=5$ square plaquettes, as shown in fig $3 \mathrm{~b}$. We thus obtain a constrained annealed average where all the relevant variables on the non connected loops inside the crosses are frozen. Since $n_{j}=16$, from (4.6) the free energy is easily evaluated, and is shown in fig 1 . The ground state energy and the residual entropy are respectively

$$
E_{a c}^{c r}(T=0)=-1.484375 \quad S_{a c}^{c r}(T=0)=0.00882 \ldots
$$

The next step is to considered the elongated cross of the type shown in fig $3 \mathrm{c}$ $\left(n_{p}=8\right.$ and $\left.n_{j}=24\right)$. In this case we get

$$
E_{a c}^{e c r}(T=0)=-1.477865 \quad S_{a c}^{e c r}(T=0)=0.0130 \ldots
$$

At increasing the size of the subsets of the decomposition, the convergence to the quenched ground state energy $E_{0}$ is rather slow as the numerical result of [11] gives $E_{0}=-1.404 \pm 0.002$ and $\left.S_{0}=0.075 \pm 0.004\right)$. However, the main qualitative feature (positive residual entropy) is reproduced by our approximations.

We have also applied our technique to the $\pm J$ spin glass in three dimensions. From a topological point of view, a $3 d$ lattice with $N$ points can be thought as the union of distinct cubes distributed in such a way that two of them have only one lattice point in common (the $3 d$ equivalent of the $2 d$ chessboard). In the thermodynamic limit their number is $\frac{1}{4} N$. Let us stress that in this case the six plaquettes of each cube represent the 'black' regions for a total of $\frac{3}{2} N$, while there are $\frac{3}{2} N$ 'white' plaquettes between cubes.

It is easy to realize that there exists a different, but equivalent, change of random variables with respect to the one previously described, which involves $n_{p}=5$ 'black' 
plaquette frustrations for each cube (the neglected plaquette must be always the same), together with other plaquette frustrations in the 'white' areas, for a total of $2 N$ independent random variables. After performing the 'white' annealed average, the problem is reduced to the calculation of the quenched free energy of a single cube. In this case we obtain a constrained annealed average of the free energy of the global system $f_{a c}^{c u b e}$, where the constraints are imposed over the products of the $J_{i j}$ 's

on all the closed loops of bonds in the $\frac{1}{4} N$ independent cubes. The resulting $f_{a c}^{c u b e}$ has a very long analytic expression and is plotted in fig 4, where it is also drawn for comparison the annealed free energy. Unfortunately, the residual entropy is still negative, thus indicating that in three dimensions more constraints are necessary to get a fair approximation of the quenched system at low temperature. However, the free energy should be a non-decreasing function of the temperature, so that the ground state energy can be estimated as the supremum of an annealed approximation although the free energy has negative derivative at low temperature [5]. Our constraints on all the loops inside the alternated cubes allows to estimate $E_{0}(d=3) \geq-1.917$ which improves the lower bounds given by the supremum of the annealed free energy, $E_{0} \geq-1.956$

\section{Constrained annealed averages on infinite loops}

The method introduced in the previous section could allow us to obtain very accurate estimates (lower bounds) of the quenched free energy. Indeed the main limitation we have met is that the number of plaquettes $n_{p}$ of the sub-system should be not too large in order to perform an analytic calculation of its quenched free energy. For instance in $2 d$ we have stopped the estimates at the elongated cross with $n_{p}=8$ plaquettes. However, we can consider infinite loops by estimating the free energy of sub-systems of infinite size only in one direction, via the Lyapunov exponent of the product of 
random transfer matrices [12].

To simplify, let us consider again the two dimensional Ising model with $J_{i j}= \pm 1$ with equal probability, although our discussion can be extended to three and higher dimensions. The idea is to find an independent decomposition of the $2 d$ lattice in strips of size $L$. We then compute the quenched free energy of the strip as the Lyapunov exponent of the infinite product of random transfer matrices of size $2^{L} \times 2^{L}$. In such a way, we automatically obtain the annealed average where the products of $J_{i j}$ 's on all the possible loops inside the strip are constrained to their quenched mean value. In particular, some of the loops are of infinite size in one direction. Our proposal requires a numerical calculation of the Lyapunov exponent, but it is superior to the direct application of the transfer matrix method [13], since it allows one to obtain lower bounds of the free energy which become more and more accurate at increasing the size $L$ of the strips, as a consequence of the standard inequalities satisfied by the constrained annealed averages.

In order to reduce the number of random variables, it is convenient to perform a gauge transformation, before computing the free energy of the spin glass. In two dimensions, we have chosen a gauge transformation which map the original system into a new one where the horizontal couplings are i.i.d. random variables $\left(J_{i j}= \pm 1\right.$ with equal probability) and the vertical couplings are positive and constant $\left(J_{i j}=1\right)$. We consider strips of $L$ spins which are parallel to the bisectrix of the lattice as shown in fig 5 , in order to obtain a convenient independent decomposition of the lattice with the properties discussed at the beginning of sect 4 . The basic cell of the strip is formed by three layers, the first and the third ones of length $L$ and the intermediate layer of length $L+1$. We denote by $\sigma$ the spins of the first layer, $\xi$ the spins of the second layer and $\eta$ the spins of the third layer. The particular form of the strip allows one to 
perform a preliminary analytic integration over the spins of intermediate layers. For instance, the case $L=3$ is illustrated in fig 5 . The partition function of that strip can be obtained by a product of transfer matrices between the first and the third spin layers:

$$
\widetilde{Z}_{N}(L=3)=\operatorname{Tr} \prod_{i=1}^{N} \mathbf{T}\left(i ; J_{1}, \cdots, J_{6}\right)
$$

where there are $2^{6}$ different random transfer matrix T. As the Boltzmann factor $\exp (-\beta H)$ of three consecutive layers of the $L=3$ strip is

$$
\begin{aligned}
& \exp \left(\sigma_{1} \xi_{1}+\sigma_{2} \xi_{2}+\sigma_{3} \xi_{3}\right) \exp \left(J_{1} \sigma_{1} \xi_{2}+J_{2} \sigma_{2} \xi_{3}+J_{3} \sigma_{3} \xi_{4}\right) \\
& \exp \left(\eta_{1} \xi_{2}+\eta_{2} \xi_{3}+\eta_{3} \xi_{4}\right) \exp \left(J_{4} \eta_{1} \xi_{1}+J_{5} \eta_{2} \xi_{2}+J_{6} \eta_{3} \xi_{3}\right)
\end{aligned}
$$

one immediately sees that, after integrating out the intermediate lay (the four $\xi$ spins), the elements of the $8 \times 8$ transfer matrices are

$$
T_{\sigma_{1}, \sigma_{2}, \sigma_{3}, \eta_{1}, \eta_{2}, \eta_{3}}=
$$

$\cosh \left(\sigma_{1}+J_{4} \eta_{1}\right) \cosh \left(J_{1} \sigma_{1}+J_{5} \eta_{2}+\sigma_{2}+\eta_{1}\right) \cosh \left(J_{2} \sigma_{2}+J_{6} \eta_{3}+\sigma_{3}+\eta_{2}\right) \cosh \left(J_{3} \sigma_{3}+\eta_{3}\right)$

The extension to a generic value of $L$ is straightforward, and one has to deal with the product of $2^{2 L}$ independent random matrices of size $2^{L} \times 2^{L}$.

The Lyapunov exponent $\lambda$ of the product of the transfer matrices is related to the quenched free energy $f_{\text {strip }}^{(L)}[12]$ by:

$$
\lambda=-n_{s} \beta f_{\text {strip }}^{(L)}
$$

A moment of reflection shows that there are $n_{s}=2 L+1$ spins and $n_{j}=4 L$ couplings, so that from (4.7) one obtains the constrained annealed free energy $f_{a c}^{(L)}$ of the total system:

$$
-\beta f_{a c}^{(L)}=\frac{\lambda-\ln 2}{2 L}
$$


The constrained free energy as function of the temperature for $L=1, L=2, L=3$ and $L=8$ is shown in fig 6 . It is evident that the difference between the constrained annealed free energy $f_{a c}^{(L)}$ and the quenched one increases at lowering the temperature, since the frustration effect becomes more and more important. In two dimensions at varying the size of the transversal length $L$ we found a monotonous convergent sequence of approximations from below to the ground state energy and to the residual entropy:

$$
\begin{array}{lll}
L=1 & E_{0}=-1.500 & S_{0}=0.00 \\
L=2 & E_{0}=-1.464 & S_{0}=0.02 \\
L=3 & E_{0}=-1.448 & S_{0}=0.03 \\
L=4 & E_{0}=-1.438 & S_{0}=0.04 \\
L=5 & E_{0}=-1.429 & S_{0}=0.05 \\
L=6 & E_{0}=-1.425 & S_{0}=0.05 \\
L=7 & E_{0}=-1.423 & S_{0}=0.05 \\
L=8 & E_{0}=-1.421 & S_{0}=0.05 \\
L=9 & E_{0}=-1.419 & S_{0}=0.06
\end{array}
$$

In conclusion, we have shown that the constrained annealing in $\pm J$ spin glasses can be performed without Lagrange multipliers, by performing quenched averages on appropriate sub-systems. However, it is an open problem to extend it to other coupling distributions such as the gaussian distribution.

\section{Acknowledgements}

GP and MS acknowledge the financial support (Iniziativa Specifica FI3) of the I.N.F.N., National Laboratories of Gran Sasso, Gruppo collegato dell'Aquila. 


\section{Appendix: How many Lagrange multipliers for the quenched free energy?}

Strictly speaking, the method of Lagrange multipliers, as formulated in [1] shows that computing an annealed average of the partition function with some constraints which hold in the thermodynamic limit is equivalent to determining the maximum of an appropriate Gibbs potential $g(\beta, \mu)$ with respect to $\mu$. Let us note that in general $\mu$ indicates a whole set of Lagrange multipliers. Actually, this method suggests a clever way to calculate a quenched average, by finding the minima of annealed models with Lagrange multipliers even in finite volume systems. However, in this case, the Lagrange multipliers do not constrain the variables $\alpha_{N}$ to their mean value. Only in the thermodynamic limit these constraints are achieved by a saddle point estimate of the annealed average of the Grand-partition function. In both cases (finite or infinite volume systems) the most interesting question is to establish how many and what quantities are necessary to reproduce a quenched average. In this appendix we give an explicit answer. We limit our considerations to the case of a single random variable $x$, since the following arguments can be easily extended to many or infinite variables. Let us suppose that $x$ is defined over a set $X$ with a probability density $p(x)$, and let $Z(x) \geq 0$ be a function of $x$ such that the quenched average $\overline{\ln Z(x)}$ exists finite. One has the following theorem:

Theorem: Assume there is a set of $n$ functions $\left\{\alpha_{1}(x), \ldots, \alpha_{n}(x)\right\}$ and a set of $n+1$ constants $\left\{c_{0}, \ldots, c_{n}\right\}$ such that:

$$
\ln Z(x)=c_{0}+\sum_{i=1}^{n} c_{i} \alpha_{i}(x)
$$

on $X$. Then the quenched average $\overline{\ln Z(x)}$ is equal to:

$$
\overline{\ln Z(x)}=\min _{\mu_{1}, \ldots, \mu_{n}} \ln \overline{Z(x) \exp \left[-\sum_{i=1}^{n} \mu_{i}\left(\alpha_{i}(x)-\overline{\alpha_{i}(x)}\right)\right]}
$$

and the minimum is reached for $\left\{\mu_{i}=c_{i}\right\}_{i=1, \ldots, n}$. 
Proof: Recalling (A1), from a direct inspection it follows that the right hand side of equation (A2) is equal to $\overline{\ln Z(x)}$ for $\left\{\mu_{i}=c_{i}\right\}_{i=1, \ldots, n}$. By virtue of the convexity of the logarithm the proof is completed. Let us stress that, in general, there is an infinite number $n$ of functions $\left\{\alpha_{i}(x)\right\}$.

This result, i.e. that a quenched average is formally equivalent to a variational annealed problem, was first established by Morita [2], using, indeed, a different variational approach with respect to relation (A2).

This simple theorem can be usefully applied in the case of finite systems with dichotomic disorder. Let us consider, for instance, a $2 d$ Ising spin glass with nearest neighbours random couplings $\left\{J_{i j}= \pm 1\right\}$. As previously seen, the partition function $Z_{N}$ of the system of $N$ spins depends only on the plaquette frustrations $\left\{\widetilde{J}_{p}^{(i)}\right\}$, which are a set of independent dichotomic $( \pm 1)$ random variables (no boundary conditions are assumed). From elementary properties of the dichotomic variables, the free energy $y_{N}=-\frac{1}{\beta N} \ln Z_{N}$ of a realization of the system can be written as:

$$
\begin{gathered}
y_{N}=c_{0}+c_{1} \frac{1}{N} \sum_{L^{(4)}} J_{p}^{(i)}+c_{2} \frac{1}{N} \sum_{L^{(6)}} J_{p}^{(i)} J_{p}^{(j)}+c_{3} \frac{1}{N} \sum_{L^{(8)}} J_{p}^{(i)} J_{p}^{(j)}+ \\
+c_{4} \frac{1}{N} \sum_{L^{(8)}} J_{p}^{(i)} J_{p}^{(j)} J_{p}^{(k)}++c_{5} \frac{1}{N} \sum_{L^{(10)}} J_{p}^{(i)} J_{p}^{(j)} J_{p}^{(k)}++c_{6} \frac{1}{N} \sum_{L^{(12)}} J_{p}^{(i)} J_{p}^{(j)} J_{p}^{(k)}+\ldots
\end{gathered}
$$

where there are different terms with the same number of plaquettes in the product, according to the length of the resulting loop built up by these plaquettes. For instance, there are three terms by the sums of products of three plaquettes, corresponding to loops of length 8,10 and 12 . Thus the quenched free energy can be recovered with an annealed average of type (A2) where every possible function

$$
\frac{1}{N} \sum_{L^{r\left(i_{1}, \ldots, i_{k}\right)}} J_{p}^{\left(i_{1}\right)} \ldots J_{p}^{\left(i_{k}\right)}
$$

appears with its Lagrange multiplier. In the thermodynamic limit $(N \rightarrow \infty)$ this means that the quenched free energy is equal to an annealed free energy where the 
frustration computed along every kind of closed loop on the lattice is constrained to its mean value.

This last statement keeps its validity for a generic distribution of the couplings, taking into account that the frustrations along the closed loops should be substituted for the more generic variables $\left\{t_{s}^{(r)}\right\}$ defined by (2.7). In other terms, the general quantity to constrain (corresponding to (A3) for the dichotomic case) is:

$$
\frac{1}{N} \sum_{L^{r)}} t_{s}^{(r)}
$$

The proof can be achieved by arguments derived from the large number law. On the contrary, for a finite size system it is not sufficient to perform an annealed average with constraints over all the quantities (A4) to recover the quenched free energy, at difference with the dichotomic case. Indeed, the $\pm J$ spin glass has a special feature, i.e., both the partition function and its logarithm can be expressed in terms of only the quantities (A3), by virtue of the dichotomic properties of the random variables, while for a generic distribution only the partition function can be written as sum of the quantities (A4). However, in principle one can get an expression of the logarithm of the partition function recurring to power series of the (A4)'s, and, as a consequence, one can find all the quantities to constrain in order to obtain the quenched free energy for a finite volume system. 


\section{References}

[1] M. Serva and G. Paladin, Gibbs thermodynamical potentials for disordered systems, Phys. Rev. Lett. 70, 105 (1993)

[2] T. Morita, Statistical mechanics of quenched solid solutions with applications to diluted alloys, J. Math. Phys. 5, 1401 (1964)

[3] R. Kühn, D. Grensing, H. Huber, Grand ensemble solution of a Grand ensemble spin glass model, Z. Phys. B 63, 447 (1986)

[4] M. F. Thorpe and D. Beeman, Thermodynamics of an Ising model with random exchange interactions, Phys. Rev. B 14, 188 (1976)

[5] G. Toulouse and J. Vannimenus, On the connection between spin glasses and gauge field theories, Phys. Rep. 67, 47 (1980)

[6] J. Deutsch and G. Paladin, The product of random matrices in a microcanonical ensemble Phys. Rev. Lett. 62, 695 (1988)

[7] G. Toulouse, Theory of the frustration effect in spin glasses, Commun. Phys. 2, $115(1977)$

[8] G. Aeppli and G. Bhanot, Ising spin gauge theory and upper marginal dimensionality for spin glasses, J. Phys. C14, L593 (1981)

[9] G. Bhanot and M. Creutz, Ising gauge theory at negative temperatures and spin glasses, Phys. Rev. B22, 3370 (1980) 
[10] S. Scarlatti, M. Serva and M. Pasquini, Large deviations for Ising spin glasses with constrained disorder, J. Stat. Phys. (submitted)

[11] L. Saul and M. Kardar, Exact integer algorithm for the two-dimensional Ising spin glass, Phys. Rev. E 48 (1993) 48

[12] A. Crisanti, G. Paladin and A. Vulpiani Products of random matrices in statistical physics (Series in Solid State Sciences 104, Springer-Verlag) (1993)

[13] H.-F. Cheung and W. McMillan Equilibrium properties of the two dimensional random $\pm J$ Ising model, J. Phys. C16, 7027 (1983) 


\section{Figure Captions}

Fig. $1 \pm J$ spin glass in $2 d$ : the annealed free energy $f_{a}$ (dashed line) and the constrained free energies $f_{a c}^{s q}$ (full line) and $f_{a c}^{c r}$ (dot-dashed line) versus temperature $T=1 / \beta$. The constraints are imposed, respectively, on $N / 2$ independent plaquettes and on $N / 8$ independent crosses of five plaquettes.

Fig. $2 \pm J$ spin glass in $2 d$ : residual frustration $\phi_{a}(3.12)$ for the annealed model and $\phi_{a c}$ (3.11) for the constrained model. The constraint is imposed on $N / 2$ independent plaquettes.

Fig. 3 Ising model with nearest neighbour interactions on a $2 d$ lattice: decompositions of the set of the couplings in independent subsets (the black areas): a) squares, b) crosses and c) elongated crosses.

Fig. $4 \pm J$ spin glass in $3 d$ : the annealed free energy $f_{a}$ (dashed line) and constrained free energy $f_{a c}^{c u b e}$ (full line) versus $T=1 / \beta$.

Fig. 5 Ising model with nearest neighbour interactions on a $2 d$ lattice:

a) decompositions of the set of the couplings into independent subsets of infinite area (the black strips) in the case $L=3$.

b) the basic cell of the strip $(L=3)$ is formed by three layers: $\sigma, \xi$ and $\eta$ spins; after the gauge transformation the vertical couplings are fixed $\left(J_{i j}=+1\right.$, full lines $)$ and the horizontal couplings are random $\left(J_{i j}= \pm 1\right.$, dashed lines).

Fig. $6 \pm J$ spin glass in $2 d$ : quenched free energy of the system obtained by a decomposition of the lattice into independent strips, as function of the temperature $T$ at different widths $L=1,2,3,8$ of the strips. 